\title{
Functional characterization of a soybean small heat shock protein involved in the resistance response to Meloidogyne javanica
}

\section{Suellen Mika Hishinuma-Silva}

Universidade Estadual de Londrina

Valéria Stefania Lopes-Caitar

University of Tennessee

Rafael Bruno Guayato Nomura

Universidade Estadual de Londrina

Bruna Caroline Sercero

Instituto Agronomico do Parana

Aline Garcia da Silva

Universidade Estadual do Norte do Parana

Mayra Costa da Cruz Gallo de Carvalho

Universidade Estadual do Norte do Parana

Ivani de Oliveira Negrão Lopes

Embrapa Soybean

Waldir Pereira Dias

Embrapa Soybean

Francismar Corrêa Marcelino-Guimaraes ( $\square$ francismar.marcelino@embrapa.br)

Embrapa Soybean https://orcid.org/0000-0002-5828-7249

Research article

Keywords: Root-Knot Nematode, HSP, Defense response, Reproduction Factor

Posted Date: December 4th, 2019

DOl: https://doi.org/10.21203/rs.2.18298/v1

License: (1) (i) This work is licensed under a Creative Commons Attribution 4.0 International License.

Read Full License 


\section{Abstract}

Background: Small heat shock proteins (sHSPs) belong to the class of molecular chaperones that respond to biotic and abiotic stresses in plants. Previous studies have identified strong induction of the GmHsp22.4 gene in response to Meloidogyne javanica nematode in resistant soybean genotypes compared to susceptible one. This study aimed to investigate the functional involvement of this small chaperone in response to $\mathrm{M}$. javanica. First, it was evaluated the activation of the promoter region by nematode's infection and the occurrence of polymorphisms between resistant and susceptible resequenced soybean accessions in the gene coding and promoter regions. Then functional analysis using Arabidopsis thaliana lines overexpressing the soybean $\mathrm{GmHsp} 22.4$ gene, and knocked out mutants were challenged to $M$. javanica's infection.

Results: High expression levels of the GFP gene marker in transformed A. thaliana plants revealed that the promoter region of GmHsp22.4 was strongly activated after nematode infection. However, structural analysis of the soybean resistant and susceptible genotypes did not detect any polymorphisms in the whole gene model, including the $2.0 \mathrm{~kb}$ promoter region. Moreover, the reproduction of the nematode was significantly reduced in plants overexpressing GmHsp22.4 gene in A. thaliana compared to the wild type. Additionally, the reproduction of $\mathrm{M}$. javanica in the A. thaliana mutants was significantly increased and was related with the structural organization of heat shock cis element (HSE) in the promoter.

Conclusions: The soybean chaperone $\mathrm{GmHsp22.4}$ is involved in the defense response to root-knot nematode M. javanica's infection in A. thaliana. The HSE in the promoter region close to the transcriptional start site (TSS) are important to GmHsp22.4 promoter activation after nematode infection. It was not possible to detect any polymorphisms occurrence in GmHsp22.4 gene between the M. javanica-resistant and susceptible soybean genotypes, so the resistance mechanism might not be related with the transcriptional regulation in the initiation process or based on sequence level on the TSS.

\section{Background}

Despite the increases in soybean productivity through genetic gains over the years, the management of pests and diseases has a significant impact on the environment related on the application of agrochemicals and production costs [1]. Among these pathogens, the phytonematodes are found in all major areas of soybean cultivation both in Brazil and worldwide. Production losses in soybean crop in Brazil caused by nematodes are estimated at R\$ 16.2 billion [2].

Several strategies have been developed to control phytonematodes, such as crop rotation, the use of nematicides and biological treatments. Additionally, transgenic approaches involving the overexpression and silencing of genes have been attempted $[3,4]$. Finally, genetic resistance has been used in breeding programs exploring genetic loci in soybean, such as the quantitative trait loci (QTLs) described in plant introductions (PIs) [5]. However, a few sources of resistance are available, limiting the development of broad durable resistance [6]. Thus, understanding the molecular mechanisms involved in the resistance 
response is an important approach for the development of biotechnological strategies for the control of these pathogens.

Chaperone proteins are present in both prokaryotes and eukaryotes and are widely distributed in several species in the plant kingdom. In plants, biotic and abiotic stresses can trigger diverse defense mechanisms, such as the activation of a group of highly conserved proteins known as heat shock proteins (HSPs) [7]. The main function of the HSPs is to act as molecular chaperones, performing maintenance of the spatial structure of other proteins that are negatively affected by changes in factors such as temperature $[8,9]$. These proteins were initially observed in the salivary glands of Drosophila spp. under heat shock stress [8]. Based on their sequence size and homology, the HSPs have been grouped into five classes: HSP60, HSP70, HSP90, HSP100 and HSP20 (or sHSP). HSP20 proteins exhibit an Nterminal hydrophobic region that is quite divergent in its sequence and length in different proteins, followed by a conserved domain of approximately 90-100 amino acid residues in the C-terminal part of the protein and a short C-terminal extension [10-13].

Despite their name, HSP20 proteins are induced not only after thermal shock but also by other abiotic stimuli, such as water deficits, heavy metals, ozone and UV radiation [14], as well as under different biotic stresses, such as nematode infection $[12,14,15]$. Studies on the functions of cytosolic HSP20 proteins have suggested that HSP20 maintains the remaining cellular proteins in an active state under stressful conditions via the linking of its dissociated dimers with denatured proteins $[10,13,16,17]$. According to this hypothesis, the heat-induced dissociation of HSP20 could lead to exposure of the hydrophobic region and, consequently, to the stabilization of the denatured proteins $[12,13]$. HSP20 then cooperates with other ATP-dependent molecular chaperones such as HSP70, HSP90, HSP100 and GroEL to refold proteins $[11,13]$. In addition, HSP20 exhibits a much higher binding stoichiometry than other molecular chaperones, leading to some speculation that HSP20 functions as a reservoir to stabilize the flow of denatured proteins in response to stress $[12,13]$.

In a previous study, Lopes et al. [18] characterized the expression profiles of 51 members of the HSP20 family in Glycine max (GmHsp20) under abiotic (heat and cold) and biotic stresses (infection by $M$. javanica) on susceptible (BRS133) and resistant (P1595099) genotypes. The expression levels of these genes were strongly dependent on the genotype under biotic stress conditions. Additionally, the exposure period (four or eight days after inoculation) played a significant role in the gene expression in both genotypes. Among the five of the 51 members of the $\mathrm{GmHsp} 20$ family that were significantly expressed under both treatments, the expression of the GmHsp22.4 (abbrev. for Glyma10g176400) gene stood out. Its relative expression level was 60 times higher in the infected resistant genotype than in the noninfected conditions. Interestingly, the authors reported the systematic occurrence of a standard organization of cis-elements in the promoter region of these $\mathrm{GmHsp20}$ genes associated with the responses of soybean to M. javanica infection.

In this study, we sought to elucidate the involvement of GmHsp22.4 in response to M. javanica infection. It was possible to verify the activity of the $\mathrm{GmHsp22.4}$ gene promoter after nematode infection in 
transgenic $A$. thaliana plants through the green fluorescent protein (GFP) marker. No single polymorphisms were detected across the whole gene coding and promoter regions of GmHsp22.4 among resequenced resistant and susceptible soybean genotypes, indicating that the regulation of $\mathrm{GmHsp22.4}$ might involve other mechanisms that are not dependent on sequence variations in the gene regulatory and coding regions. For the functional characterization, $A$. thaliana seeds were subjected to Agrobacterium-mediated transformation to achieve overexpression of theGmHsp22.4 gene, resulting in

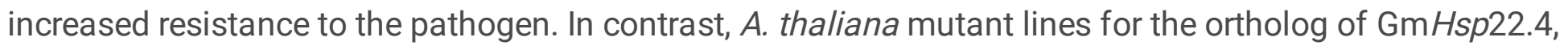
At4g10250, presented a reduction in resistance, confirming that the gene is required to mediate defense against $M$. javanica. The levels of resistance were dependent of the T-DNA insertion in the promoter region, being reduced in events interrupted in HSE elements close to the TSS.

\section{Results}

\section{The GmHsp22.4 promoter is strongly induced by $M$. javanica in Arabidopsis roots}

To investigate the ability of the $\mathrm{GmHsp22.4}$ promoter to respond to $M$. javanica infection, the GmHsp22.4 promoter sequence was fused with the coding region (CDS) of GFP and introduced into $A$. thaliana. The levels of fluorescence in the roots were measured after infection with $M$. javanica in four different events by comparing inoculated and noninoculated plants at 9 dai. As expected, the average GFP activity was increased in all events tested (PGmHsp22.4-3, 4, 6 and 12) (Figure 1).. No activity was detected in the control plants.

\section{Structural and polymorphism analysis of $\mathrm{GmHsp22.4} \mathrm{in} \mathrm{M}$. javanica resistant and susceptible genotypes}

GmHsp22.4 presented a total length of 1,158 bp with no intron region, resulting in a potential encoded protein of 159 aa. The orthologous gene in $A$. thaliana exhibited a similar organization, presenting $74.6 \%$

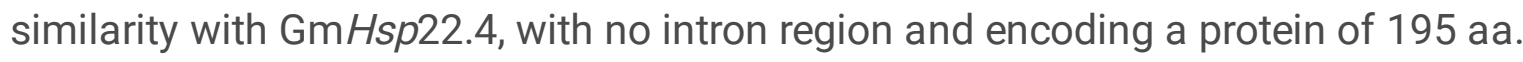

Based on the resequencing data obtained by Santos et al. [19] of 21 soybean accessions, a potential promoter region spanning $2.0 \mathrm{~kb}$ from the transcription start site and the complete transcriptional region (including exons and UTRs) were compared between soybean genotypes [6] that are resistant and susceptible to $M$. javanica. The GmHsp22.4 gene was highly conserved in all genotypes evaluated, and no changes were detected among the sequences. Similarly, the promoter region showed no changes, except for a substitution in the $-1,229$ position (A/T) upstream of the transcription start site, but it was not possible to associate this substitution with resistance to the pathogen, as it was randomly distributed among the genotypes. 


\section{Overexpression of $\mathrm{GmHsp22.4} \mathrm{increases} \mathrm{resistance} \mathrm{to} \mathrm{root-knot}$ nematodes in Arabidopsis}

Agrobacterium tumefaciens carrying the GmHsp22.4_pH7WG2D construct was used to transform $A$. thaliana. Stable $A$. thaliana plants overexpressing the coding region of $\mathrm{GmHsp22.4}$ under $35 \mathrm{~S}$ promoter control were obtained by floral dip transformation. Positive events were selected and confirmed by the detection of eGFP fluorescence in $A$. thaliana roots. Strong green fluorescence in the roots associated with three homozygous events in the T4 generation, but not in the wild type (WT), demonstrated the success of transformation with GmHsp22.4 (Figure 2)..

The transformed plants were evaluated regarding their root morphology due to the overexpression of

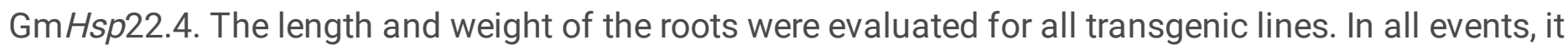
was possible to detect an average root length of $8.0 \mathrm{~cm}$, and no significant difference compared with the wild type was detected (Additional File 1).. Similarly, root weight showed no difference between the events and was approximately $60 \mathrm{mg}$ (Additional File 1)..

The expression levels of the $\mathrm{GmHsp22.4}$ gene in the 14 transgenic plants for each event were quantified by RT-qPCR. As expected, high levels of gene expression were detected in $A$. thaliana plants transformed with the target gene regulated by the 35S promoter (Figure 3A).. The highest expression was observed in the $\mathrm{Gm} H s p 22.4-8-\mathrm{OE}$ line, followed by the $\mathrm{GmHsp22.4-6-OE}$ and $\mathrm{Gm} H s p 22.4-4-\mathrm{OE}$ events, respectively.

The effect of $\mathrm{GmHsp22.4}$ overexpression in $A$. thaliana transgenic plants against $M$. javanica infection was examined by counting the number of $M$. javanica females on plant roots compared to wild-type plants at 22 day after inoculation (dai) (Figure 3B).. Among the three tested homozygous events, a significant reduction in the number of females was observed for the $\mathrm{GmHsp22.4-6-OE} \mathrm{(82 \% )} \mathrm{and}$ GmHsp22.4-8-OE (42\%) events compared to the WT (OE). The GmHsp22.4-4-OE event did not present a significant reduction in the number of $M$. javanica females (Figure 3B)..

\section{Knockout of the GmHsp22.4 orthologue in Arabidopsis compromises the defense response to $M$. javanica}

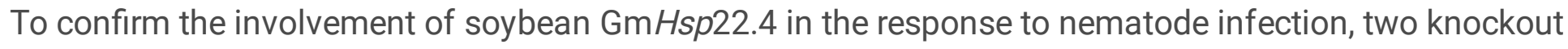
A. thaliana mutants for the orthologous gene were tested against pathogen infection. The athsp22.0-1 and athsp22.0-2 mutants presented a T-DNA inserted in the promoter at -191 pb or in the 5' UTR at +149 $\mathrm{pb}$, respectively. The homozygous lines of the mutants were selected by PCR (Additional File 2 and Additional File 5)..

The effects of the mutations on plant growth and development were verified. No significant differences were detected for the morphological parameters evaluated between transgenic and wild-type plants. The average root length of athsp22.0-1 and athsp22.0-2 was $11.4 \mathrm{~cm}$, while that of the WT was $11.2 \mathrm{~cm}$ (Additional File 3).. Similar results were found when root weight was evaluated; for athsp22.0-1 and 
athsp22.0-2, the values were 835.3 and 938.2 milligrams, respectively, while that of WT was 976.4 milligrams (Additional File 3).. The number of leaves and the shoot weight did not present any significant differences (Additional File 3).. Thus, the results showed that in both mutant events, it was not possible to identify significant effects on the development of the plants compared to the wild-type plants.

Finally, the effect of the mutations on the nematode's ability to infect and multiply on $A$. thaliana plants were evaluated. The number of females associated with both events at 45 dai showed a significant increase of approximately $60 \%$ compared to the WT (Figure $4 A$ ). Similarly, the number of juveniles/eggs was higher in the $A$. thaliana athsp22.0-2 mutant, at 2,631.15, while for the athsp22.0-1 mutant and the control, the number were 1,636.29 and 964,686, respectively (Figure 4B)..

Based on the results, the athsp22.0-1 and athsp22.0-2 A. thaliana mutants showed increased susceptibility to M. javanica. Similarly, the athsp22.0-2 mutant exhibited an increase in the number of eggs and juveniles compared to wild-type plants, which was significant at the $5 \%$ level.

\section{Analysis of cis-elements in the 5 'UTR and promoter region of the Arabidopsis At4g10250 gene}

Based on the analysis of the promoter region of At4g10250, we identified a TA-rich sequence at the position of +114 bp and TATA boxes at $+79,-146,-165$ and -456 bp of the transcription start site (TSS). CAAT box elements were found in the sequence at positions $+182,+173,+71,+5,-2,-121,-126,-165$, $-181,-310$ and -355 bp of the TSS. The HSEs, which are recognized and activated by the heat shock factors (HSF) transcription factor [20], were observed at six different positions, $+166,+48,-97,-408,-$ 462 and -467 bp of the TSS. In contrast to the other elements, a W-box was located in the negative strand at the -303 bp position (Figure 5)..

In the T-DNA insertion lines, it was possible to identify the location of the T-DNA insert between the CAATbox and W-box cis-elements in the WiscDsLox489_492E13 event, while the T-DNA insertion site in the 5' UTR of GK-265F12-014990 was located between the cis-element HSE and TA-rich (Figure 5)..

\section{Discussion}

In this study, we examined the role of the $\mathrm{GmHsp22.4}$ gene in the root-knot nematode resistance response in detail. This gene was selected because, in a previous study, it was found to be strongly expressed only in an $M$. javanica-resistant genotype at 8 dai, whereas in a susceptible genotype, the expression of this gene was repressed [18]. Based on this finding, we hypothesized that these chaperones play a differential role in the infection response during the infection cycle of the nematode related to the type of damage and alterations caused by this pathogen in host roots [18].

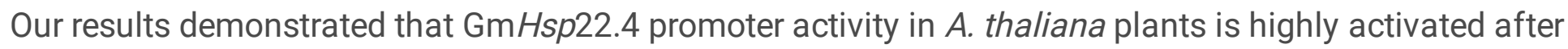
nematode infection, confirming the importance of this gene in the response to $M$. javanica. The 
GmHsp22.4 promoter presents a structural organization in which CAAT boxes are located immediately upstream of HSE elements, while a W-box is located further upstream of the HSEs, as previously described by Lopes-Caitar [18], and it is potentially recognized by an HSF. As expected, promotor induction was $98 \%$ greater in the infected Arabidopsis transgenic plants than in the noninfected ones, as revealed by GFP fluorescence marker (Figure 1)..

Although we demonstrated the activity of the promoter of the GmHsp22.4 in M. javanica responses, we could not demonstrate how such different levels of $\mathrm{GmHsp22.4}$ transcripts and the resultant contrasting phenotypes between the resistant and susceptible soybean accessions are explained the gene sequence level. Our analysis of 21 resequenced soybean accessions did not detect any variation in the promoter, 5'UTR, 3'UTR or exons of the GmHsp22.4 gene that correlated with the phenotype. The whole gene and promoter regions were identical between the resistant and susceptible materials evaluated.

The differential regulation of the expression of $\mathrm{GmHsp22.4}$ between the resistant and susceptible genotypes was in accordance with the results observed by Fuganti et al. [21]. These authors mapped a QTL related to root-knot nematode resistance in a population derived from the resistant source PI 595099 between two microsatellite markers, Satt 144 and Soy HSP 176. The Soy HSP 176 marker was identified as being located in a region containing the GmHsp17.6-L gene. GmHsp17.6-L expression levels were subsequently shown to be differentially regulated between resistant and susceptible individuals from the mapping population and were induced only in resistant individuals. When Fuganti et al. [15] analyzed the

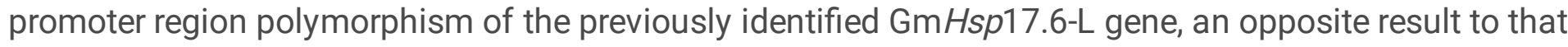
observed in our study was revealed, since the authors detected a greater number of AT (n) repeats in the resistant genotype compared to the susceptible genotype, whereas in our results, the number of AT (n) repeats in GmHsp22.4 remained constant in the different genotypes [15]. So, in our analysis it was not possible observe any correlation of AT(n) repetitions and promoter activity of GmHsp22.4.

The occurrence of enhancer regions that interfere with the promoter regions of other genes in their vicinity or over long distances, interfering with their regulation, might be a possible explanation, but our data cannot confirm this hypothesis. In addition, we speculate that epigenetic regulation, which cannot be detected at a sequence level, might be an alternative explanation for the results. This mechanism has been reported to be involved in resistance to cyst nematodes [22]. Similarly, Rambani et al. [23] evaluated the impacts of soybean cyst nematode (SCN) infection on DNA methylation patterns in the root tissues from susceptible soybean plants. Interestingly, the authors identified 447 soybean genes that were differentially methylated in the promoter region, comparing samples inoculated with a cyst nematode versus those that were not inoculated.

This differential methylation may be related to the accessibility of transcription factors to cis-elements, thereby regulating the transcriptional activity of genes responsive to the nematodes, as described by Zhou et al. [24]. In their study, it was observed that the HsfA1a transcription factor played an essential role in the activation of the resistance response mediated by the Ml-1.2 gene. In addition, HsfA1a interfered with the activation of HSP90, which participates in Mi-1.2 gene activation. This observation 
was made after the silencing of HsfA1 in tomato plants. This scenario may be an alternative explanation

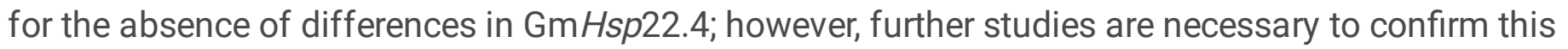
hypothesis.

To obtain a better understanding of the roles of these chaperones in the resistance response to root-knot nematodes, we overexpressed the soybean gene in $A$. thaliana plants and studied two DNA insertion lines in which the orthologous genes were knocked out. In our study, two events involving constitutive

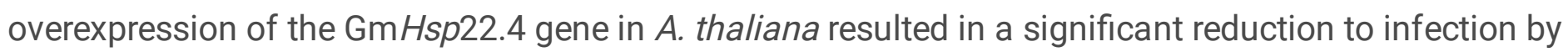
the root-knot nematode compared to untransformed plants. Increasing the expression of the heat shock gene in A. thaliana plant roots led to reductions in the numbers of females by $82 \%$ and $42 \%$ for the two events (Figure 3B).. Similarly, when the orthologous gene was knocked out in Arabidopsis plants, we observed an increase in susceptibility of $60 \%$ (Figure 4 )..

Arabidopsis presents 19 genes encoding Hsp20s, grouped into 12 subfamilies based on their subcellular localization and homology [18], and the transcript of the $A$. thaliana gene model orthologous to

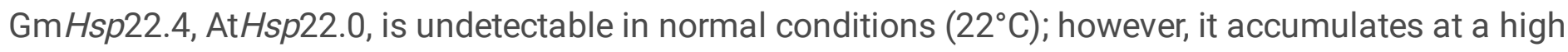
level in response to heat stress $\left(38^{\circ} \mathrm{C}\right)$ [25]. To date, there is no available evidence of the activation of At $H s p 22.0$ under biotic stress conditions, such as nematode infection. The mode of action of these proteins after stress is becoming clearer, suggesting that HSP2O effectively captures protein folding intermediates that are prone to aggregation and maintains them in a suitable conformation for refolding. Thus, HSP20 has an oligomeric structure that interacts with nonnative proteins. This structure exists in two states: an inactive low-affinity state and an active high-affinity state. After heat stress, HSP2O is activated to its high-affinity state and becomes competent to associate with denatured proteins, forming a stable complex. This enables efficient prevention of irreversible protein aggregation. However, the release of these proteins from the complexes that are formed requires the cooperation of ATP-dependent HSPs, such as HSP70-HSP40 or HSP100. Although these other proteins may act directly on the aggregated proteins, the presence of HSP20 increases the efficiency of the process [26].

Based on this analysis, we suggest that $\mathrm{GmHsp22.4}$ is involved in nematode infection responses, where one of the hypotheses is that it acts as the first line of cellular defense by capturing unfolded proteins, reducing the size of protein aggregates; thus, more binding sites are generated, and with the assistance of ATP-dependent HSP70 and HSP100, aggregation is reversed and refolding is facilitated [27]. The high transcript levels of $\mathrm{GmHsp22.4}$ upon its overexpression in transformed plants may have improved the stability of plant proteins in the reticulum, favoring the defense response, or have maintained the supply of active chaperones in the plant during pathogen infection.

The involvement of different soybean small heat shock proteins in nematode resistance to the cyst nematode $H$. glycines was previously demonstrated by Kandoth et al. [28]. In this study, a comparison of the gene expression profile of the genotypes harboring Rhg 1 locus was performed in soybean plants at the developmental stage of the syncytium through laser capture microdissection (LCM) and subsequent microarray analysis. These results indicated the presence of at least three $H s p 20$ coding genes with 
upregulated profiles in the syncytium of the resistant near-isogenic lines (NILs) derived from a cross between the susceptible cv Evans and the resistant PI 209332.

The role of $H s p 20$ related to its nematode-responsive promoter activity has been described in rice by Escobar et al [29], who characterized the involvement of the soybean HsHsp17.4 gene in the response to infection by M. incognita. Upon the binding of the HsHsp17.4 promoter region to the $\beta$-glucuronidase (GUS) marker gene, they observed that the promoter induced the expression of GUS and, consequently, $10 \%$ galls in the roots were stained after 1 to 3 days of infection with $M$. incognita, with the staining reaching 50 to $70 \%$ at 17 to 20 days postinfection. Mutations in the $83 \mathrm{bp}$ region upstream from the transcription start point are determinant for promoter activation in the nematode response [29]. Therefore, the athsp22.0-2 mutant, which exhibits a T-DNA insertion at $+149 \mathrm{bp}$, could affect this crucial region where the binding of heat shock transcription factors occurs, also confirming the importance of this HSE element close to TSS in the GmHsp22.4 ortholog after nematode infection.

Similarly, Barcala et al. [14] described the importance of combinations and/or specific sequences of HSEs for regulation in different situations. Functional analysis of the promoter regions of HaHsp17.6G1 and

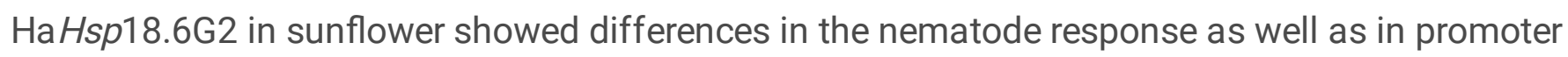
structure. HaHsp17.6G1 was not induced after nematode infection, while HaHsp18.6G2 was induced in giant cells. The structural analysis demonstrated the presence of two HSEs in the HaHsp18.6G2 promoter, one of which was proximal, while the other was distant. In contrast, only one HSE was observed in the HaHsp17.6G1 promoter, in a distant region. It was also found that the CAAT box element in HaHsp18.6G2 was located immediately upstream of and between the HSEs, while in the HaHsp17.6G1 promoter, it was located downstream of the HSE.

Interestingly, it was possible to observe an organization of the soybean gene promoters responsive to nematode infection similar to that of the cis-elements of the At $4 \mathrm{~g} 10250$ promoter as previously described by Lopes-Caitar [18]. The promoters of the soybean Hsp20 genes responsible for $M$. javanica infection presented two CAAT elements in the region containing the HSE element in their structures, while the Wbox was located farther away [18]. On the other hand, it was not possible to observe the occurrence of an HSE element within the -83 bp region from the TSS, but it was closer located at -97 bp position from the TSS. Thus, the similar structural organization among Hps22.4 among soybean and Arabidopsis could also reflect functional conservation.

\section{Conclusions}

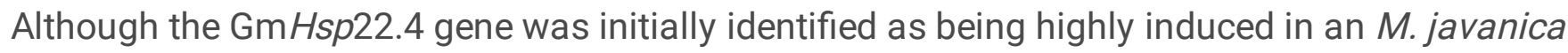
nematode-resistant soybean genotype and repressed in a susceptible genotype, no differences in polymorphism were observed in either the promoter or coding regions between the soybean resistant and

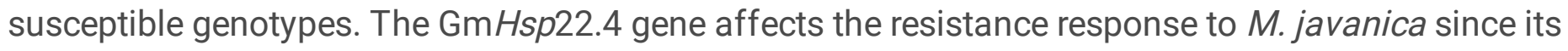
knockout increases the susceptibility of the inoculated plants by $60 \%$, and its overexpression reduces the infective potential of nematodes by up to $82 \%$. The promoter of the $\mathrm{GmHsp} 22.4$ gene is induced in 
response to infection with $M$. javanica at 9 dai, but as there is no difference between the susceptible and resistant plant promoters. The promoter activation is dependent of its structure, and the presence of HSE close to the TSS is essential.

\section{Materials And Methods}

\section{Plant Materials}

Seeds of soybean accession PI 595099 used in this study were provided by Soybean Germoplasm Bank from Embrapa Soybean. Arabidopsis thaliana seeds of two independent lines from a single knockout mutant of AtHsp22.0 were obtained from the Arabidopsis Biological Resource CenterABRC)WiscDsLox489_492E13 with stock number N858258 for athsp22.0-1 and GK-265F12014990 with stock number N335081 for athsp22.0-2).

\section{Polymorphism analysis of the GmHsp22.4 gene}

Resequencing data of 21 soybean genotypes were obtained from Santos et al. [19]. The fasta file for the nucleotide sequence spanning the interval of $41,003,835$ to $41,006,424$ was uploaded.

The phenotypic responses of the materials to M. javanica infection were obtained at the Embrapa SojaNematology laboratory [6], with BRS Valiosa RR, BRSGO 8360, CD 201, MG/BR46 Conquista, PI 595099 and Paraná being classified as resistant cultivars and Anta 82, BR 16, BRS 232, BRS 360RR, BRS 361, BRS Sambaíba, BRSGO Chapadões, BRSMT Pintado, BRSMT Uirapuru, FT Abyara, FT Cristalina, IAS 5, NA 5909 RG, P98Y11 and Williams82 as susceptible.

The nucleotide sequences of all 21 genotypes were evaluated with Integrative Genomics Viewer (IGV) [30].

\section{Cloning of the $\mathrm{GmHsp22.4} \mathrm{gene} \mathrm{from} \mathrm{soybean}$}

The DNA of the M. javanica nematode-resistant soybean genotype PI 595099 was extracted from leaf tissue according to the technique described by Doyle and Doyle [31] and used for the amplification of the CDS region with the primers CDSGmHsp22.4-F and CDSGmHsp22.4-R, while the promoter region was amplified with the primers PGmHsp22.4-F and PGmHsp22.4-R (Additional File 5).. The PCR fragments were purified using the Wizard $\circledast S V$ gel and PCR Clean-UP System (Promega).

The fragments were introduced into an input vector with the PCR ${ }^{\text {TM }} 8 / G W / T O P O \circledast$ TA Cloning ${ }^{\circledR}$ Kit (2012), which was then used for the transformation of cells of the electrocompetent strain Escherichia coli DH5a by electroporation. The selection of the transformed clones was performed through the use of the antibiotic hygromycin at a final concentration of $100 \mu \mathrm{g} . \mathrm{mL}-1$ and enzyme analysis, while the 
recombinants and the clones containing the fragments in the expected orientation were determined by restriction reactions. The plasmid DNA from the clones containing the promoter and CDS regions of $\mathrm{GmHsp22.4}$ in the expected orientation were used in a recombination reaction with the vector pHGWFS7_P [32] for promoter analyses (Figure 6A) and the vector pH7WG2D_CDS for coding region analyses (Figure 6B) [32].

\section{A. tumefaciens GV3101 and plant transformation}

The plasmids pHGWFS7 and pH7WG2D containing the correctly cloned fragments of interest were used to transform A. tumefaciens GV3101 strains by electroporation at 2,2 kV, $25 \mu \mathrm{F}$, with 1 wrist controller at 200 or 400 ohms. Plates containing YEP medium with gentamicin and hygromycin were incubated overnight at $28^{\circ} \mathrm{C}$. For the confirmation of positive bacterial clones, PCR was performed using the primer set PGmHsp22.4-F and PGmHsp22.4-R for the promoter and the primer set pH7WG2D-F and pH7WG2D-R for the CDS (Additional File 5).. The recombinant bacteria were used to transform the $A$. thaliana Columbia (Col-0) ecotype, using the floral dip method [33]. The selection of transformed seeds in T0 and the subsequent T2, T3 and T4 generations was performed in \medium containing 1 / 2x MS medium (Sigma Chemicals $n^{\circ} \mathrm{m}-5519$ ), $0.8 \%$ agar (Sigma Chemicals $\mathrm{n}^{\circ} \mathrm{A}-1296$ ), and $15 \mu \mathrm{g} / \mathrm{ml}-1$ of hygromycin. Transformants were identified as hygromycin-resistant seedlings when they did not present growth retardation. Positive events were confirmed via PCR (Additional File 4) and then propagated until the T4 lineage to be used in subsequent experiments.

\section{Molecular analysis of putative transgenic plants}

The A. thaliana plants transformed with $\mathrm{GmHsp22.4}$ and selected with the antibiotic hygromycin at $15 \mu \mathrm{g}$ $\mathrm{mL}^{-1}$ were confirmed by PCR using genomic DNA extracted from the roots (Additional File 4).. The primer pairs PGmHsp22.4-F and PGmHsp22.4-R (promoter region) or pH7WG2D-F and pH7WG2D-R (coding region) were used for PCR according to the instructions of the Taq DNA Polymerase Invitrogen Kit. DNA extracted from untransformed plants was used as a negative sample. The PCR conditions for the promoter region were $95^{\circ} \mathrm{C}$ for 4 minutes, followed by 35 cycles of $95^{\circ} \mathrm{C}$ for $30 \mathrm{~s}, 56^{\circ} \mathrm{C}$ for $30 \mathrm{~s}$ and $72^{\circ} \mathrm{C}$ for $45 \mathrm{~s}$ and one additional step of $72^{\circ} \mathrm{C}$ for 5 minutes.

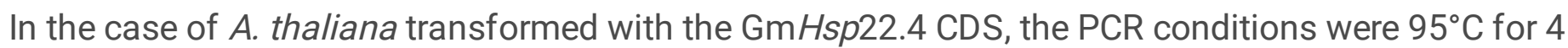
minutes, followed by 35 cycles of $95^{\circ} \mathrm{C}$ for 30 seconds, $62^{\circ} \mathrm{C}$ for 30 seconds and $72^{\circ} \mathrm{C}$ for 45 seconds, and an additional step at $72^{\circ} \mathrm{C}$ for 5 minutes.

Both of the products of amplification were analyzed by conventional electrophoresis, and translucent and photodocumentador (Loccus) equipment was used to acquire and store the images.

\section{Detection of GFP activity under M. javanica infection}


The A. thaliana transgenic lines PGmHsp22.4-3, PGmHsp22.4-4, PGmHsp22.4-6, and PGmHsp22.412, harboring PGmHsp22.4:GFP were used to determine the promoter activity of GmHsp22.4 on the basis of green fluorescence intensity. The evaluation was performed in the roots of plants infected with $400 \mathrm{~J}_{2}$ $M$. javanica after 9 day of inoculation or left uninfected [18]. The experiment was conducted in a CRD with three plants and four independent transformation events. Images of roots expressing eGFP were captured with a 5.0 megapixel camera connected to a Zeiss Axio Scope A1 compound microscope (Zeiss Corporation) and processed with MOTIC software (version 2.0). Quantitative analysis of fluorescence levels was performed by using the Adobe ${ }^{\circledR}$ Photoshop ${ }^{\circledR}$ CS6 $13.0 \times 32$ program. The statistical analysis was performed with SAS [34] using ANOVA, and the means were compared at the $5 \%$ level of significance by Student's $t$-test.

\section{Bioassays with $M$. javanica}

For the functional analysis, 14 nontransformed plants and plants transformed with the GmHsp22.4 CDS, overexpressing the GFP marker, were selected for a nematode bioassay conducted in a completely randomized design (CRD). The population of $M$. javanica was multiplied, and the roots were challenged with $400 \mathrm{M}$. javanica $\mathrm{J}_{2}$ per plant. The inocula were pipetted through a small open hole next to the root of the plant.

After 22 days of inoculation [35], the roots were collected and weighed individually. Evaluation of the number of females of $M$. javanica was performed by counting the nematodes stained with acid fuchsine according to [36]. All parameters evaluated were compared between wild-type plants and those transformed with the GmHsp22.4 gene. The statistical analysis was performed using the Statistical Analysis System (SAS) [34] using ANCOVA, and the means were compared at the $5 \%$ level of significance with Student's $t$-test.

\section{Quantification of $\mathrm{GmHsp22.4} \mathrm{transcript} \mathrm{levels}$}

Total RNA was extracted using the TRIzol reagent ( $1 \mathrm{~mL} / 100 \mathrm{mg}$ tissue) as recommended by the manufacturer (Invitrogen). After extraction, the RNA samples were treated with deoxyribonuclease I (Kit Invitrogen - DNase I) according to the manufacturer's recommendations to eliminate any DNA molecules present in the sample. The treated RNA was employed in the cDNA synthesis step using the SuperScript III Kit (Invitrogen) as recommended by the manufacturer. Real-time PCR was conducted in a StepOnePlus ${ }^{\text {TM }}$ System thermocycler (Thermo Fisher Scientific) using the SYBR ${ }^{\circledR}$ Green PCR Master Mix kit (Applied Biosystems) according to the manufacturer's instructions. For normalization, the AT_Act (actin At3g18780) gene was employed, which was amplified with the primers AT_Act-F and AT_Act-R, while wild-type control plants were employed as calibrators for each corresponding treatment. Target gene expression levels determined using the primer set RT-GmHsp22.4-F and RT-GmHsp22.4-R (Additional File 5) were calculated by the REST program. 


\section{Assay of T-DNA insertion mutants}

Two independent lines from a single knockout mutant of AtHsp22.0 were obtained from the Arabidopsis Biological Resource Center (ABRC) (WiscDsLox489_492E13 with stock number N858258 for athsp22.0-1 and GK-265F12-014990 with stock number N335081 for athsp22.0-2). Sequence analysis revealed that the T-DNA of athsp22.0-1 is inserted at $191 \mathrm{bp}$ upstream relative to the transcript initiation site and that of athsp22.0-2 at 149 bp downstream (Figure 7).. Regarding the size of the T-DNA insert, that of athsp22.0-1 was 8954 bp in length, and that of athsp22.0-2 was $6498 \mathrm{bp}$ in length.

The homozygosity of these two mutations was verified using the primer pairs LP/RP and BP1/RP for athsp22.0-1 and LP/RP and BP2/RP for athsp22.0-2 (Additional File 5).. One of the amplification reactions involved the use of the LP (left border) and RP (right border) primers (Additional File 5),, which surround the region flanking the insert, so it was only possible to verify amplification in the control plants, while amplification was not observed in the mutant plants due to the size of the insert. On the other hand, in the reaction using the primer RP (right border) as well as the primers BP1 and BP2 (Additional File 5)," which anneal to the T-DNA in the promoter and exons, respectively, resulted in amplification only in mutant plants, as the control plants did not present the insertion.

After 45 days, the total number of females and eggs/juveniles stained with fuchsine acid [36] was evaluated according to Coolen and D' Herde [37]. The two evaluated parameters were compared between the control plants and the mutants under a CRD. Statistical analysis was performed with SAS [34] using ANOVA at the $5 \%$ significance level by Student's $t$-test.

\section{Cis-element identification in the At4g10250 promoter}

Putative cis-elements from the 500 bp region upstream of the transcript start site of the At $4 \mathrm{~g} 10250$ gene were characterized by bioinformatics using the PlantCARE [38] and AthaMap [39] databases.

\section{Declarations}

Declarations

Ethics approval and consent to participate

Not applicable.

Consent for publication

Not applicable.

Funding 
This study was supported by the National Institute of Science and Technology - INCT Plant Stress-CNPq, National Council for the Improvement of Higher Education (CAPES), and EMBRAPA - Brazilian Federal Government.

\section{Availability of data and materials}

All data generated during this study are included the in the manuscript or supplementary information files.

\section{Competing interests}

The authors declare that they have no competing interests.

\section{Authors' contributions}

SMHS, VSLC and FCMG planned and designed the study. VSLC performed the computational analysis. SMHS executed the experiments, generated the figures and drafted the manuscript. RBGN, BCS and AGS also contributed to the execution of the experiments and sample preparation. WPD provided the nematode material and contributed to the orientation of the nematode experiment. IONL performed the statistical data analysis. VSLC, RBGN, MCCGC, FCMG and IONL contributed to the discussion of the manuscript. All authors read and approved the final manuscript.

\section{Acknowledgements}

We wish to thank our laboratory coworkers from EMBRAPA Soybean for their support and the National Council for the Improvement of Higher Education (CAPES) for a scholarship.

\section{Authors Information}

Department of Biochemistry and Biotechnology, Londrina State University, Londrina, Brazil

Suellen Mika Hishinuma-Silva, Rafael Bruno Guayato Nomura

Department of Plant Sciences, University of Tennessee, Knoxville, TN

Valéria Stefania Lopes-Caitar

Department of Production and Plant Protection, Agronomic Institute of Paraná-IAPAR, Londrina, Brazil

Bruna Caroline Sercero

Northern Paraná State University, Bandeirantes, Brazil

Aline Garcia da Silva, Mayra Costa da Cruz Gallo De Carvalho 
Department of Plant Biotechnology, Brazilian Agricultural Research Corporation EMBRAPA Soybean, Londrina, PR, Brazil

Ivani de Oliveira Negrão Lopes ${ }^{5}$, Waldir Pereira Dias ${ }^{5}$ and Francismar Corrêa Marcelino-Guimarães ${ }^{5}$

\section{List of Additional Files}

Additional File 1. Phenotype of Arabidopsis thaliana overexpressing GmHsp22.4 and WT Arabidopsis thaliana after Meloidogyne javanica inoculation $(n=14)$. ( $A$ ) Root length and $(B)$ Root weight. Data are expressed as the mean \pm standard error of the mean. No significant differences were found between event and WT ( $t$-test, $p \leq 5 \%$ ) (JPEG $1.5 \mathrm{MB})$.

Additional File 2. Molecular characterization of the Arabidopsis thaliana knockout mutants, athsp22.01 and athsp22.0-2. (A) PCR amplification of the athsp22.0-1 mutant, with the LP, left primer. RP, right primer. BP1, primer of T-DNA athsp22.0-1 left border. (B) PCR amplification of the athsp22.0-2 mutant, with the LP, left primer. RP, right primer. BP2, primer of T-DNA athsp22.0-2 left border (JPEG $66 \mathrm{~KB}$ ).

Additional File 3. Morphological characterization of athsp22.0-1, athsp22.0-2 and WT $(n=20)$. (A) Root length in $\mathrm{cm}$. (B) Root weight in $\mathrm{mg}$. (C) Number of leaves. (D) Fresh aerial part weight in $\mathrm{mg}$. Data are expressed as the mean \pm standard error of the mean. No significant differences were found between event and WT ( $t$-test, $p \leq 5 \%$ ) (JPEG $2.8 \mathrm{MB})$.

Additional File 4. Molecular confirmation of the GmHsp22.4 promoter and CDS inserts in Arabidopsis thaliana. (A) PCR amplification of the promoter $\mathrm{GmHsp22.4}$ region of different events and WT. (B) PCR amplification of the CDS GmHsp22.4 region of different events and WT. In both transformations, the images shown are representative of about 14 biological replicates for each condition. In the insertion with promoter region the size of the amplicon was $1076 \mathrm{pb}$, the insertion of the CDS region was $1331 \mathrm{pb} . \mathrm{M}$ indicates the $1 \mathrm{~kb}$ plus DNA Ladder (JPEG $145 \mathrm{~KB}$ ).

Additional File 5. Primers used in this study (XLSX 10.0 KB).

\section{Abbreviations}

ABRC: Arabidopsis Biological Resource Center

bp: base pair

CaMV: Cauliflower mosaic virus

CDS: Coding region

dai: Day after inoculation

GFP: Green fluorescent protein 
GUS: $\beta$-Glucuronidase

HSE: Heat shock element

HSF: Transcription factors

Inoc: inoculated

Mock: Not inoculated

LCM: Laser capture microdissection

QTL: Quantitative trait loci

SAS: Statistical Analysis System

SCN: Soybean cyst nematode

sHSP: Small heat shock protein

TSS: Transcription start site

WT: Wild type;

\section{References}

1. Lima FS, Correa VR, Nogueira SR, Santos PR: Nematodes affecting soybean and sustainable practices for their management. Soybean-the basis of yield, biomass and productivity 2017:95110.

2. Machado ACZ: Praga custa R\$ 35 bilhões ao agronegócio brasileiro. In: Revista Agrícola. vol. 24. Pedrinhas Paulista; 2015.

3. Lopes M: Aspectos histopatológicos e mudanças na expressão de genes em genótipos de soja resistente, durante a interação com Meloidogyne javanica. 2009.

4. Kim K-S, Vuong TD, Qiu D, Robbins RT, Shannon JG, Li Z, Nguyen HT: Advancements in breeding, genetics, and genomics for resistance to three nematode species in soybean. Theoretical and applied genetics 2016, 129(12):2295-2311.

5. André LdL, Sonah H, Dias WP, Marcelino-Guimarães FC, Belzile F, Abdelnoor RV: Genome-wide association study for resistance to the southern root-knot nematode (Meloidogyne incognita) in soybean. Molecular breeding 2017, 37(12):148.

6. de produção de soja-Região T: Central do Brasil 2014. Londrina: Embrapa Soja 2013.

7. Al-Whaibi MH: Plant heat-shock proteins: a mini review. Journal of King Saud University-Science 2011, 23(2):139-150. 
8. de Souza Freitas WE, de Oliveira Fernandes PL, Leite GA, Dantas JIA, Pontes CA, de Carvalho CAC: Ação das Proteínas de Choque Térmico em frutos. AGROPECUÁRIA CIENTíFICA NO SEMIÁRIDO 2012, 8(2):24-31.

9. Lopes-Caitar VS, Silva SMH, Marcelino-Guimaraes FC: Plant small heat shock proteins and its interactions with biotic stress. In: Heat shock proteins and plants. Springer; 2016: 19-39.

10. Haslbeck M: sHsps and their role in the chaperone network. Cellular and Molecular Life Sciences CMLS 2002, 59(10):1649-1657.

11. Kotak S, Larkindale J, Lee U, von Koskull-Döring P, Vierling E, Scharf K-D: Complexity of the heat stress response in plants. Current opinion in plant biology 2007, 10(3):310-316.

12. Van Ooijen G, Lukasik E, Van Den Burg HA, Vossen JH, Cornelissen BJ, Takken FL: The small heat shock protein $20 \mathrm{RSI} 2$ interacts with and is required for stability and function of tomato resistance protein I-2. The Plant Journal 2010, 63(4):563-572.

13. Xu Y, Zhan C, Huang B: Heat shock proteins in association with heat tolerance in grasses. International journal of proteomics 2011, 2011.

14. Barcala M, García A, Cubas P, Almoguera C, Jordano J, Fenoll C, Escobar C: Distinct heat-shock element arrangements that mediate the heat shock, but not the late-embryogenesis induction of small heat-shock proteins, correlate with promoter activation in root-knot nematode feeding cells. Plant molecular biology 2008, 66(1-2):151-164.

15. Fuganti R, Machado MdFPd, Lopes VS, Silva JFV, Arias CAA, Marin SRR, Binneck E, Abdelnoor RV, Marcelino FC, Nepomuceno AL: Size of A T (BioMed Research International 2010, 2010.

16. Lee GJ, Roseman AM, Saibil HR, Vierling E: A small heat shock protein stably binds heat-denatured model substrates and can maintain a substrate in a folding-competent state. The EMBO journal 1997, 16(3):659-671.

17. Liberek K, Lewandowska A, Ziętkiewicz S: Chaperones in control of protein disaggregation. The EMBO journal 2008, 27(2):328-335.

18. Lopes-Caitar VS, de Carvalho MC, Darben LM, Kuwahara MK, Nepomuceno AL, Dias WP, Abdelnoor RV, Marcelino-Guimarães FC: Genome-wide analysis of the Hsp20 gene family in soybean: comprehensive sequence, genomic organization and expression profile analysis under abiotic and biotic stresses. BMC genomics 2013, 14(1):577.

19. dos Santos JVM, Valliyodan B, Joshi T, Khan SM, Liu Y, Wang J, Vuong TD, de Oliveira MF, MarcelinoGuimarães FC, Xu D: Evaluation of genetic variation among Brazilian soybean cultivars through genome resequencing. BMC genomics 2016, 17(1):110.

20. Scharf K-D, Berberich T, Ebersberger I, Nover L: The plant heat stress transcription factor (Hsf) family: structure, function and evolution. Biochimica et Biophysica Acta (BBA)-Gene Regulatory Mechanisms 2012, 1819(2):104-119.

21. Fuganti R, Beneventi MA, Silva JFV, Arias C, Marin SR, Binneck E, Nepomuceno AL: Identificação de marcadores moleculares de microssatélites para seleção de genótipos de soja resistentes a Meloidogyne javanica. Nematologia Brasileira 2004, 28(2):125-130. 
22. Cook DE, Bayless AM, Wang K, Guo X, Song Q, Jiang J, Bent AF: Distinct copy number, coding sequence, and locus methylation patterns underlie Rhg1-mediated soybean resistance to soybean cyst nematode. Plant physiology 2014, 165(2):630-647.

23. Rambani A, Rice JH, Liu J, Lane T, Ranjan P, Mazarei M, Pantalone V, Stewart CN, Staton M, Hewezi T: The methylome of soybean roots during the compatible interaction with the soybean cyst nematode. Plant Physiology 2015, 168(4):1364-1377.

24. Zhou J, Xu X, Cao J, Yin L, Xia X, Shi K, Zhou Y, Yu J: HsfA1a is essential for Mi-1.2-mediated resistance to Meloidogyne incognita, and regulates Wfi1 transcription and $\mathrm{H} 2 \mathrm{O} 2$ production. Plant Physiology 2018:pp. 01281.02017.

25. Helm KW, Schmeits J, Vierling E: An endomembrane-localized small heat-shock protein from Arabidopsis thaliana. Plant physiology 1995, 107(1):287.

26. Haslbeck M, Franzmann T, Weinfurtner D, Buchner J: Some like it hot: the structure and function of small heat-shock proteins. Nature structural \& molecular biology 2005, 12(10):842-846.

27. Mogk A, Ruger-Herreros C, Bukau B: Cellular Functions and Mechanisms of Action of Small Heat Shock Proteins. Annual review of microbiology 2019, 73.

28. Kandoth PK, Ithal N, Recknor J, Maier T, Nettleton D, Baum TJ, Mitchum MG: The soybean Rhg1 locus for resistance to the soybean cyst nematode Heterodera glycines regulates the expression of a large number of stress-and defense-related genes in degenerating feeding cells. Plant physiology 2011, 155(4):1960-1975.

29. Escobar C, Barcala M, Portillo M, Almoguera C, Jordano J, Fenoll C: Induction of the Hahsp17. 7G4 promoter by root-knot nematodes: involvement of heat-shock elements in promoter activity in giant cells. Molecular plant-microbe interactions 2003, 16(12):1062-1068.

30. Thorvaldsdóttir H, Robinson JT, Mesirov JP: Integrative Genomics Viewer (IGV): high-performance genomics data visualization and exploration. Briefings in bioinformatics 2013, 14(2):178-192.

31. Doyle JJ, Doyle JL: Isolation ofplant DNA from fresh tissue. Focus 1990, 12(13):39-40.

32. Karimi M, Inzé D, Depicker A: GATEWAY ${ }^{\mathrm{TM}}$ vectors for Agrobacterium-mediated plant transformation. Trends in plant science 2002, 7(5):193-195.

33. Zhang X, Henriques R, Lin S-S, Niu Q-W, Chua N-H: Agrobacterium-mediated transformation of Arabidopsis thaliana using the floral dip method. Nature protocols 2006, 1(2):641.

34. Sas S, Guide SUs: Version 9.1. SAS Institute Inc, Cary, NC 2003.

35. Curtis RH, Powers SJ, Napier J, Matthes MC: The Arabidopsis F-box/Kelch-repeat protein At2g44130 is upregulated in giant cells and promotes nematode susceptibility. Molecular plant-microbe interactions 2013, 26(1):36-43.

36. Taylor A, Sasser J: Biology, identification and control of root-knot nematodes. North Carolina State University Graphics 1978, 111.

37. Coolen W, d'Herde C: A method for the quantitative extraction of nematodes from plant tissue. A method for the quantitative extraction of nematodes from plant tissue 1972. 
38. Lescot M, Déhais P, Thijs G, Marchal K, Moreau Y, Van de Peer Y, Rouzé P, Rombauts S: PlantCARE, a database of plant cis-acting regulatory elements and a portal to tools for in silico analysis of promoter sequences. Nucleic acids research 2002, 30(1):325-327.

39. Steffens NO, Galuschka C, Schindler M, BuÈlow L, Hehl R: AthaMap: an online resource for in silico transcription factor binding sites in the Arabidopsis thaliana genome. Nucleic acids research 2004, 32(suppl_1):D368-D372.

\section{Figures}

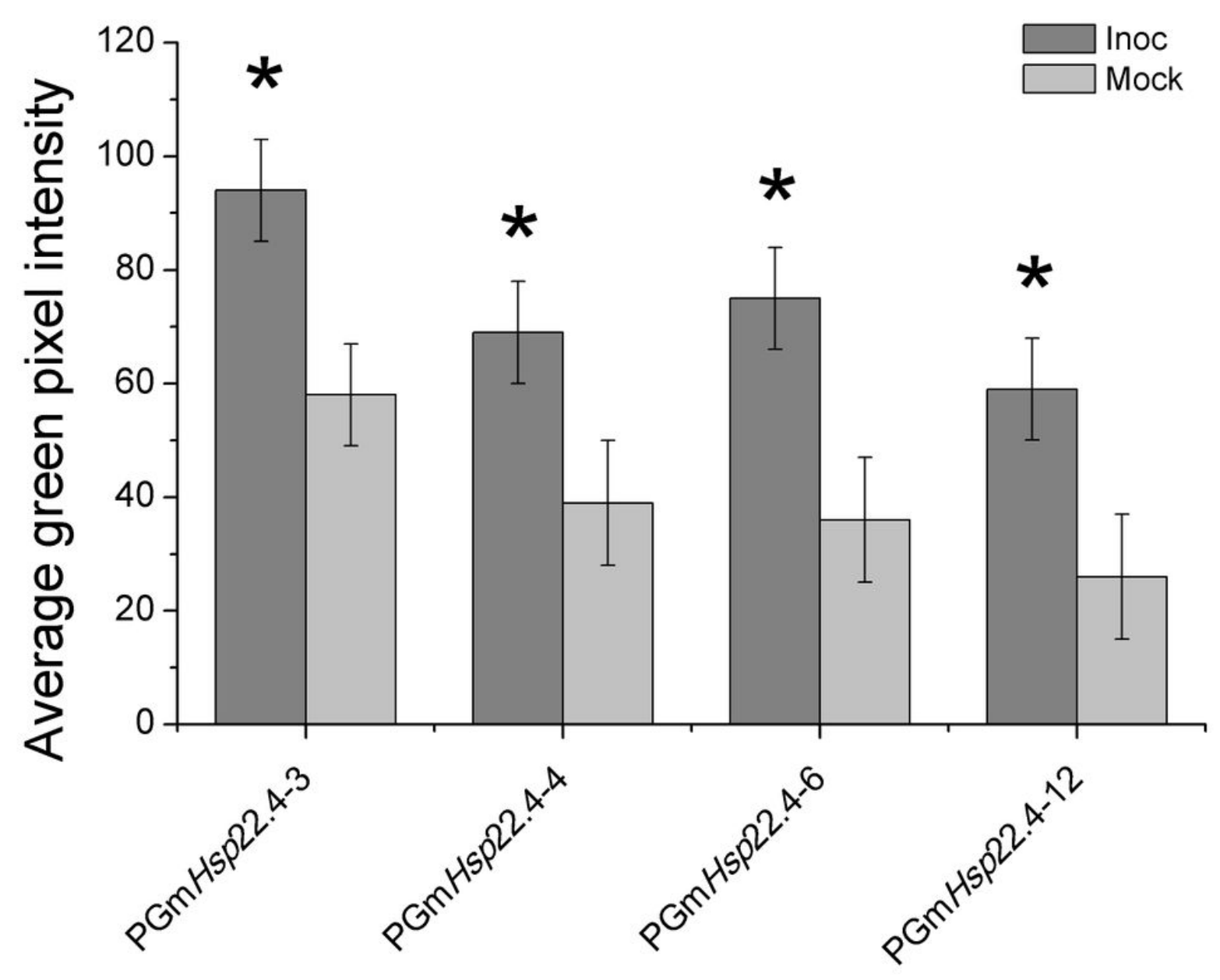

Figure 1

Expression of GFP protein in transgenic Arabidopsis thaliana roots. Arabidopsis thaliana roots of 4 stable transgenic events were observed and photographed under a fluorescence microscope after 9 days after inoculation in inoculated (inoc) or not inoculated (mock) with Meloidogyne javanica. Green fluorescence indicates expression from the promoter and induction of the GFP marker. The data shown are representative of three biological replicates for each condition (inoc and mock). Fluorescence intensity 
was analyzed with the Adobe ${ }^{\circledR}$ Photoshop ${ }^{\circledR}$ CS6 13.0 x 32 software program. * indicates statistical significance at the $5 \%$ level (t-test) compared to the mock control.

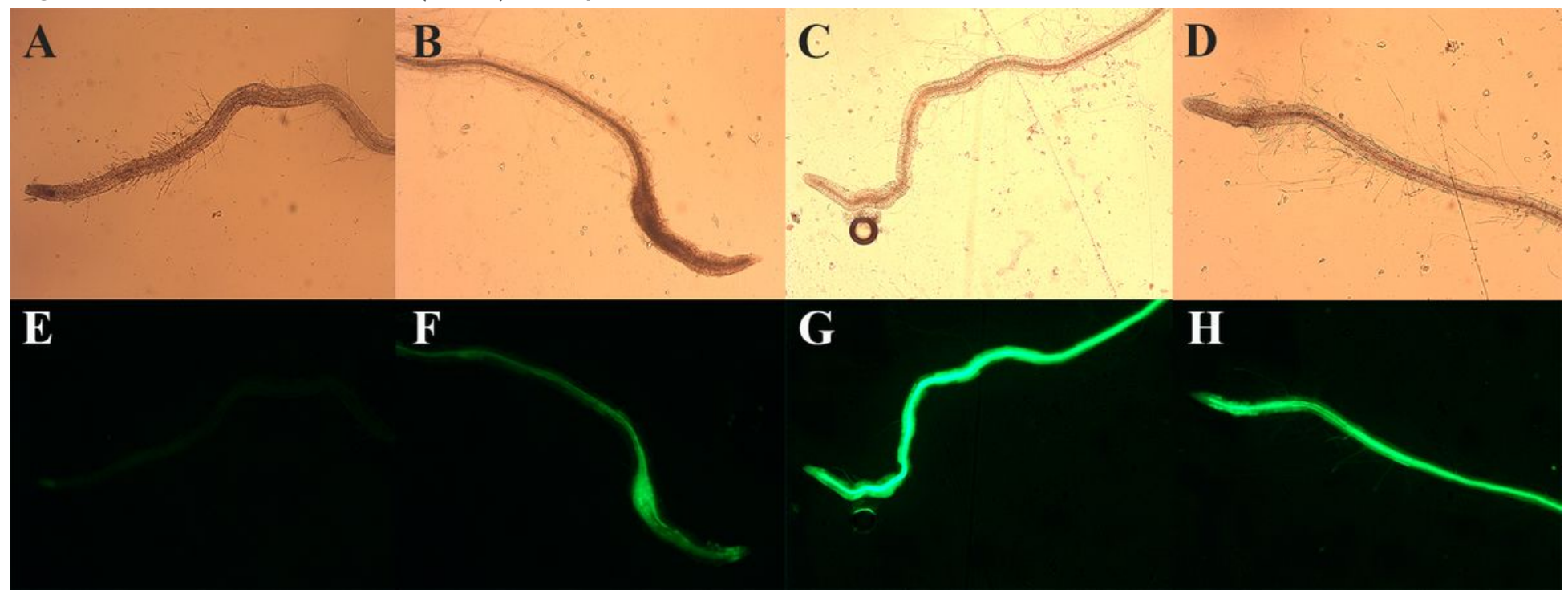

\section{Figure 2}

GFP expression in transgenic Arabidopsis thaliana roots overexpressing GmHsp22.4. The roots were observed and photographed under a Zeiss Axio Scope A1 optical microscope using a common light with no filter (A-D) and a GFP filter (E-H). (A) WT (OE) no filter. (B) GmHsp22.4-4-OE no filter. (C) GmHsp22.4-6OE no filter. (D) GmHsp22.4-8-OE no filter. (E) WT (OE) GFP filter. (F) GmHsp22.4-4-OE GFP filter. (G) GmHsp22.4-6-OE GFP filter. (H) GmHsp22.4-8-OE GFP filter.
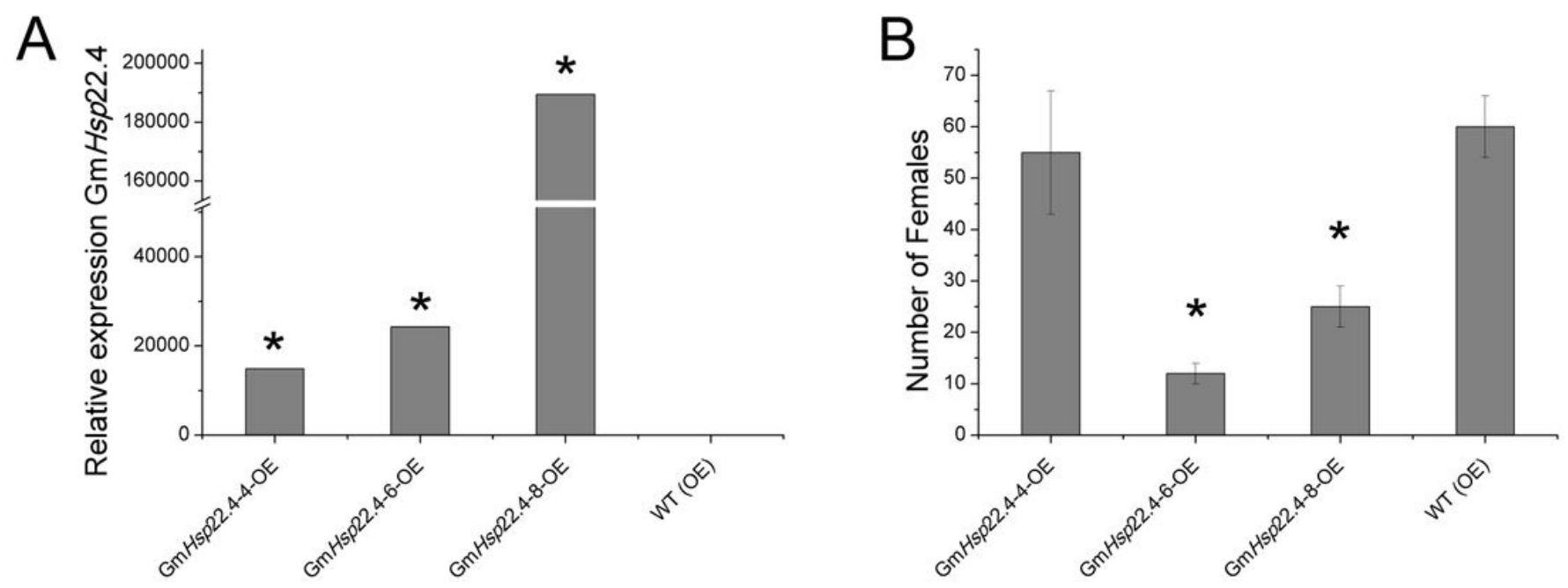

\section{Figure 3}

Evaluation of expression and numbers of Meloidogyne javanica females. (A) Relative expression of GmHsp22.4 in transformed and WT Arabidopsis thaliana plants infected with M. javanica. (B) Number of M. javanica females at 22 days after inoculation in GmHsp22.4-overexpressing and WT A. thaliana $(n=14)$. Data are expressed as the mean \pm standard error of the mean. A p-value $\leq 0,05$. * indicates statistical significance at the $5 \%$ level (t-test) compared to WT. 

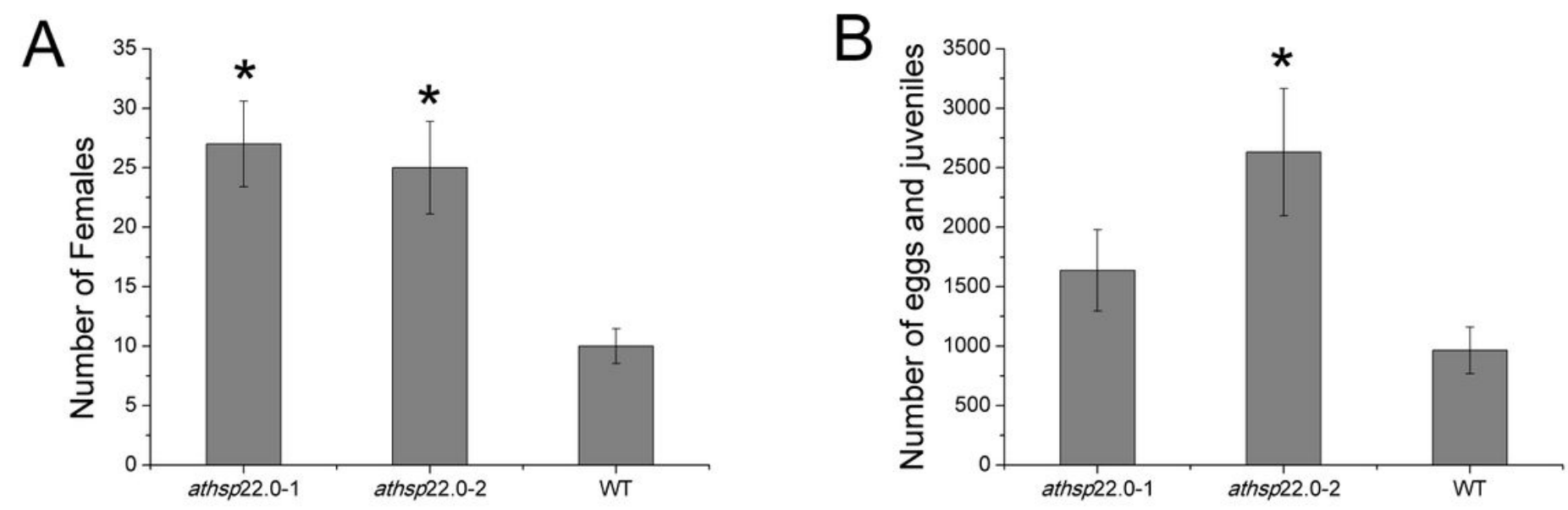

\section{Figure 4}

Resistance of the knockout mutant. athsp22.0-1 and athsp22.0-2 plants were compared to WT plants under infection with Meloidogyne javanica $(n=20)$. (A) Number of female nematodes per plant at 45 day after inoculation (dai). (B) Number of eggs / juveniles per plant at 45 dai. Data are expressed as the mean \pm standard error of the mean. ${ }^{*}$ indicates statistical significance at the $5 \%$ level (t-test) compared to WT.

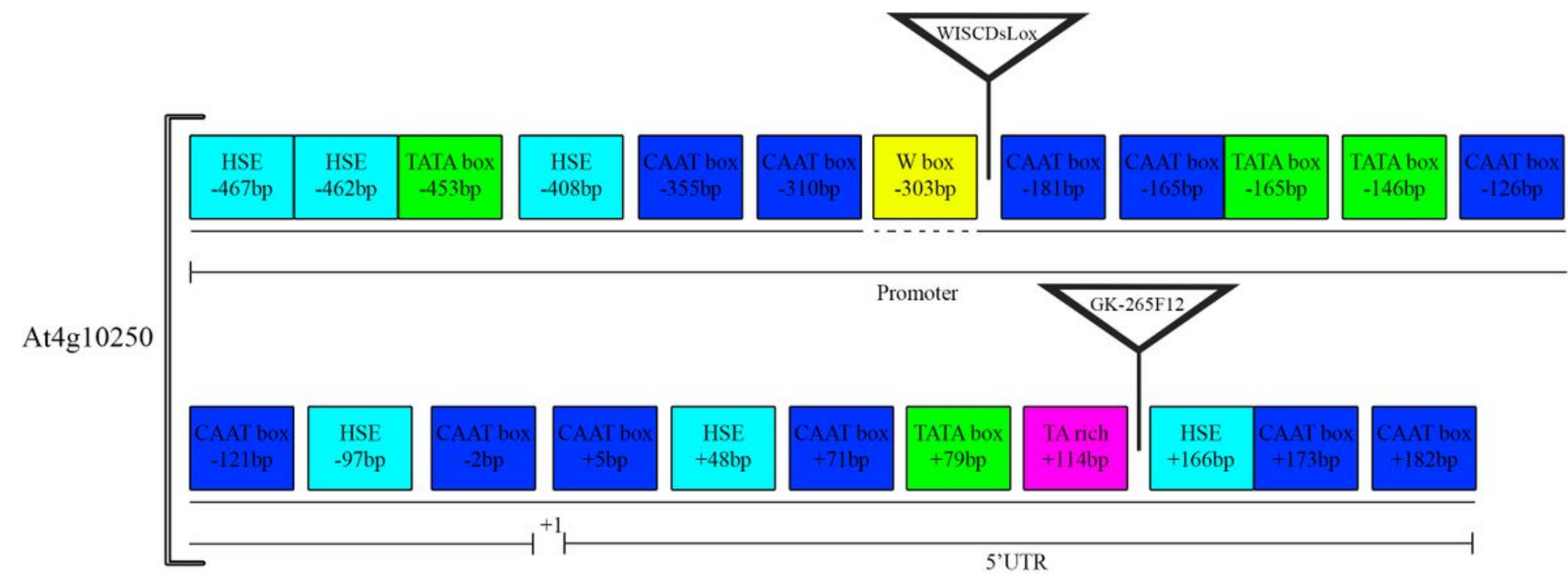

\section{Figure 5}

Schematic map of the locations of different cis elements in the At4g10250 gene. (A) Cis-element of the promoter region, (B) cis-element of the $5^{\prime}$ UTR. PlantCARE and AthaMap were used to analyze the region $500 \mathrm{bp}$ upstream of the transcription start site. The dark blue boxes represent CAAT boxes; the light blue boxes represent HSEs; the green boxes represent TATA boxes; the pink box represents the TA-rich element; and the yellow box represents the W-box. *dotted line: reverse strand. The WiscDsLox represent the T-DNA insertion site in the promoter region and 265F12 in 5' UTR region. 
A

pHGWFS7:GmHsp22.4_P

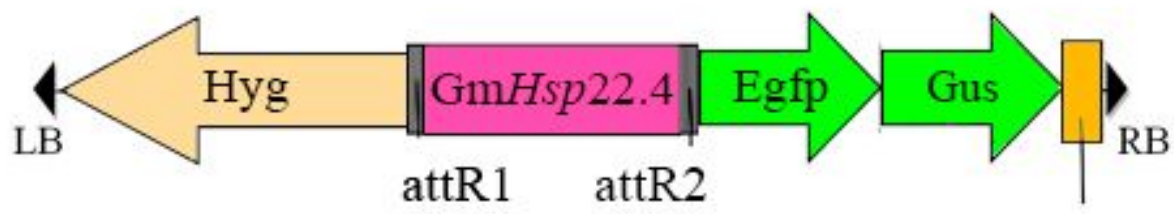

$\mathrm{T} 35 \mathrm{~S}$

B

pH7WG2D:GmHsp22.4_CDS

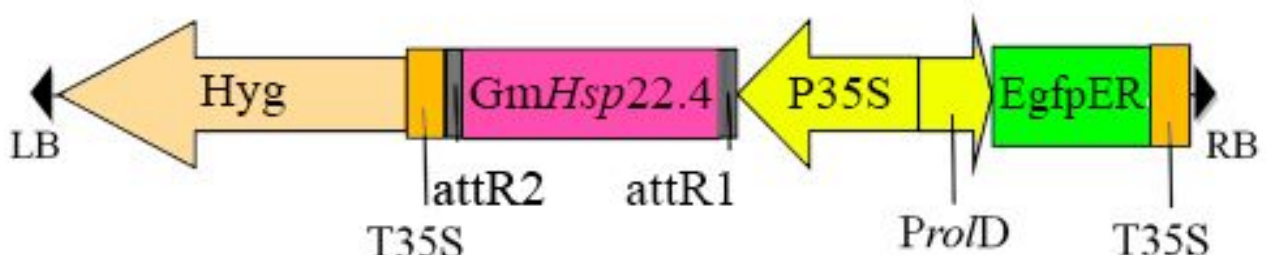

Figure 6

Scheme of the T-DNA regions of binary vectors. The vectors were used for GmHsp22.4_CDS overexpression and analysis of GmHsp22.4_P promoter activity in Arabidopsis. (A) Construct used for promoter analysis, pHGWFS7: GmHsp22.4_P. (B) Overexpression construct, pH7WG2D: GmHsp22.4_CDS. RB (RightBorder) and LB (LeftBorder), used for the transformation of plants. p35S, cauliflower mosaic virus (CaMV) 35S promoter, a highly active promoter in plants. attR1 and attR2, recombination sites. T35S, cauliflower mosaic virus terminator. Egfp encodes green fluorescence protein (GFP). Gus encodes the $\beta$-glucuronidase protein. Hyg indicates resistance to the antibiotic hygromycin. 
athsp22.0-1

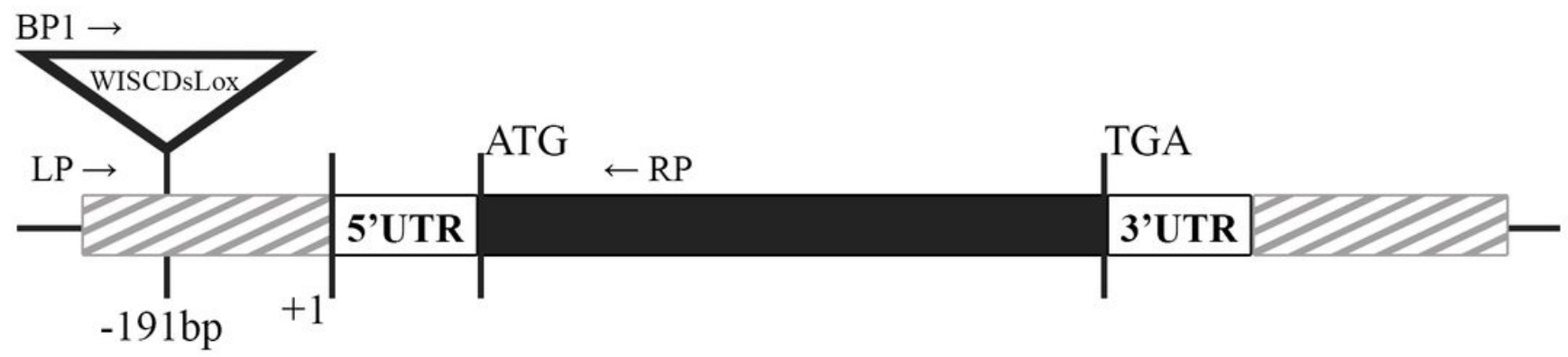

athsp22.0-2

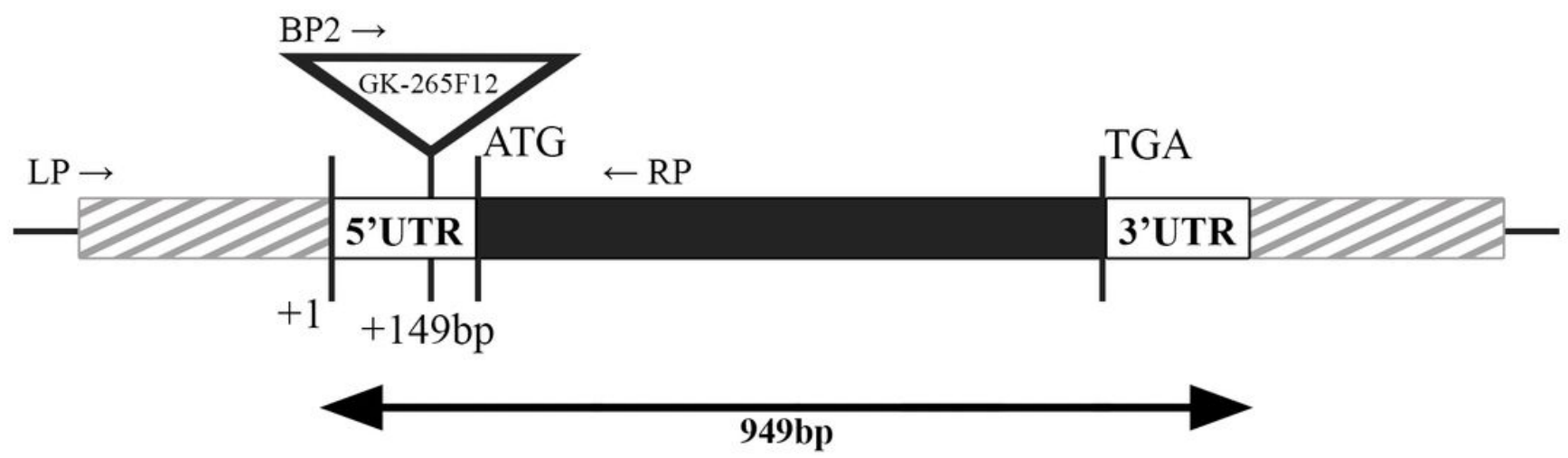

Figure 7

A scheme of the A. thaliana AtHsp22.0 gene. Dark boxes represent the coding region, and striped gray boxes represent the promoter and terminator sequences. T-DNA insertion sites are indicated for both mutations (WiscDsLox for athsp22.0-1 and GK_265F12 for athsp22.0-2), and LP, RP, BP1 and BP2 indicate the localizations of the primers used for genotyping (Additional File 5).

\section{Supplementary Files}

This is a list of supplementary files associated with this preprint. Click to download.

- AdFile3.jpg

- AdFile2.jpg

- AdFile4.jpg

- AdFile1.jpg

- AdFile5.xlsx 